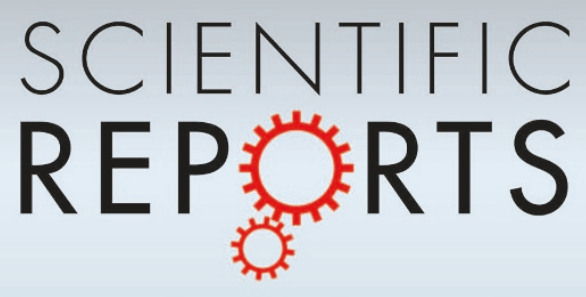

SUBJECT AREAS: NANOBIOTECHNOLOGY

IMMUNOLOGY

CELL SIGNALLING

NANOPARTICLES

Received

14 May 2012

Accepted

6 July 2012

Published

26 July 2012

Correspondence and requests for materials should be addressed to N.H. (HANAGATA. Nobutaka@nims.go.

\title{
Binding Mode of $\mathrm{CpG}$
} Oligodeoxynucleotides to Nanoparticles Regulates Bifurcated Cytokine induction via Toll-like Receptor 9

\author{
Shanmugavel Chinnathambi' ${ }^{1}$ Song Chen ${ }^{2}$, Singaravelu Ganesan' \& Nobutaka Hanagata ${ }^{3,4}$
}

\begin{abstract}
'Department of Medical Physics, Anna University, Chennai 600-025, India, ${ }^{2}$ JSPS Research Fellow, 8 Ichibancho, Chiyoda-ku, Tokyo 102-8472, Japan, ${ }^{3}$ Nanotechnology Innovation Station, National Institute for Materials Science, 1-2-1 Sengen, Tsukuba, Ibaraki 305-0047, Japan, ${ }^{4}$ Graduate School of Life Science, Hokkaido University, N1 OW8, Kita-ku, Sapporo 060-08 12, Japan.
\end{abstract}

The interaction of cytosine-phosphate-guanine oligodeoxynucleotides (CpG ODNs) with Toll-like receptor 9 (TLR9) activates the immune system. Multimeric class A CpG ODNs induce interferon- $\alpha$ (IFN- $\alpha$ ) and, to a lesser extent, interleukin-6. By contrast, monomeric class B CpG ODNs induce interleukin-6 but not IFN- $\alpha$. This difference suggests that the multimerization of CpG ODN molecules is a key factor in IFN- $\alpha$ induction. We multimerized class B CpG ODN2006x3-PD molecules that consist entirely of a phosphodiester backbone onto quantum dot silicon nanoparticles with various binding modes. Herein, we present the binding mode-dependent bifurcation of cytokine induction and discuss its possible mechanism of CpG ODN and TLR9 interaction. Our discoveries also suggest that nanoparticles play roles in not only delivery of CpG ODNs but also control of CpG ODN activity.

ligodeoxynucleotides (ODNs) that contain unmethylated cytosine-phosphate-guanine (CpG) motifs can stimulate the immune system via interaction with the pattern-recognition receptor Toll-like receptor 9 (TLR9) $)^{1-3}$. In humans, TLR9 is expressed in B cells and plasmacytoid dendritic cells (pDCs) ${ }^{4}$. TLR9 activation in B cells induces proinflammatory cytokines-including interleukin (IL)-6, IL-10, and IL-12through the signal-transduction pathways involved in nuclear factor- $\kappa \mathrm{B}^{5-7}$, whereas in $\mathrm{pDCs}$ it induces type I interferons (IFNs), tissue necrosis factor- $\alpha$, IL-6, IL-12, and IFN $\gamma$-inducible $10-\mathrm{kDa}$ protein ${ }^{3,8-13}$. The potential of CPG ODNs to stimulate the immune system via activation of TLR9 can be applied to infectious disease and allergy treatments and cancer therapy ${ }^{14-17}$.

CpG ODNs are divided into 4 classes according to their sequence properties. Class A and class B CpG ODNs have been well characterized. Those in class A have a palindromic structure consisting of a phosphodiester backbone at the center of the sequence and poly-G motifs with a phosphorothioate backbone at each end ${ }^{3,18,19}$. Class B CpG ODNs have a linear structure consisting entirely of a phosphorothioate backbone ${ }^{3,19-21}$. IFN- $\alpha$ is induced by class A, but not by class $\mathrm{B}, \mathrm{CpG} O \mathrm{ODNs}^{18}$. By contrast, class $\mathrm{B} C \mathrm{CpG}$ ODNs can proliferate and activate $\mathrm{B}$ cells, leading to IL-6 production. Conversely, class A CpG ODNs have a lower potential to induce IL- ${ }^{20}$. Class A and class B CPG ODNs contain a partial or complete phosphorothioate backbone, which improves resistance to nuclease degradation ${ }^{22-24}$. However, side effects are cause for concern ${ }^{25-30}$. We recently developed a nucleaseresistant CpG ODN consisting entirely of a phosphodiester backbone ${ }^{31}$. This novel CpG ODN, referred to as CpG ODN2006x3-PD, has a linear structure. Similar to class B CpG ODNs, CpG ODN2006x3-PD induces IL-6 but not IFN- $\alpha$.

Under certain physiological conditions, class A CPG ODNs form nanometer-sized multimers via self-assembly because of their palindromic and poly-G sequences ${ }^{32}$. By contrast, class B CpG ODNs lack the potential to form such higher-order multimers. IFN- $\alpha$ induction by class-A CpG ODNs is thought to result from the formation of multimers, the average length of which has been demonstrated to be $\sim 100 \mathrm{~nm}^{32,33}$. Interestingly, IFN- $\alpha$ production has been observed when class B CpG ODNs are loaded onto 180 -nm polystyrene nanoparticles (NPs) ${ }^{32}$, suggesting that NPs can alter the cytokine induction mediated by the interaction of class B CpG ODNs and TLR9. However, the mechanism by which class-B CpG ODNs acquire the potential to induce IFN- $\alpha$ remains unclear. 
In the present study, we aimed to clarify this mechanism. First, we investigated whether class B CpG ODNs loaded onto NPs smaller than the multimers formed by class A CpG ODNs can stimulate IFN$\alpha$ induction. Second, we examined whether IFN- $\alpha$ is induced by class B CpG ODNs remaining on the surface of the NPs or by free CpG ODNs released from the NPs. Third, we evaluated whether IFN- $\alpha$ induction is affected by the binding mode of class B CpG ODNs on the NPs. For these investigations, we prepared silicon NPs ( $\mathrm{Si}-\mathrm{NPs}$ ) with an average diameter of $3.4 \mathrm{~nm}$ because silicon has a low inherent toxicity $^{34}$. The Si-NPs displayed a substantial photoluminescence (PL) quantum yield in the visible region. Generally, polycations such as polyethyleneimine and poly-L-lysine are used to modify NPs to bind negatively charged DNA electrostatically. However, polycations have been reported to result in nonspecific binding of negatively charged molecules and promote the formation of NP aggregates ${ }^{35-37}$, which are thought to cause side effects. The toxicity of polycations likely affects cytokine induction in the immune system as well as the interaction between CpG ODNs and TLR9. Thus, we modified the SiNP surface with allylamine to ensure a positive charge and electrostatically bind them to CpG ODN molecules. Furthermore, alternative surface modification of Si-NPs was performed by introducing maleimide into the allylamine amino group to cross-link CpG ODN molecules onto Si-NP covalently. Herein we demonstrate that these binding modes significantly affect the bifurcation of cytokine induction. Our findings also demonstrate the simultaneous induction of IFN- $\alpha$ and IL- 6 using CpG ODN2006x3-PD molecules with different binding modes on Si-NPs.

\section{Results}

Characterization of positively charged Si-NPs. To examine the electrostatic binding of negatively charged CpG ODN molecules to Si-NPs, we synthesized hydrogen-terminated Si-NPs and modified their surfaces with allylamine, which has a positively charged amino group. To confirm successful modification, we examined the surface state using Fourier transform infrared spectra (FT-IR) and proton nuclear magnetic resonance ( ${ }^{1} \mathrm{H}$ NMR). Peaks at $1254 \mathrm{~cm}^{-1}$ and $1468 \mathrm{~cm}^{-1}$ in the FT-IR spectra (Fig. 1a), attributed to vibrational scissoring and symmetric bending of $\mathrm{Si}-\mathrm{CH}_{2}$, clearly indicated the presence of allylamine. An N-H stretching band between $3500 \mathrm{~cm}^{-1}$ and $3655 \mathrm{~cm}^{-1}$, an N-H scissoring peak at $1627 \mathrm{~cm}^{-1}$, and $\mathrm{C}-\mathrm{H}$ stretching peaks at $2851 \mathrm{~cm}^{-1}$ and $2923 \mathrm{~cm}^{-1}$ were also observed. Compared with $\mathrm{SiO}_{2}$, the surface-modified Si-NPs showed lowermagnitude peaks between $1000 \mathrm{~cm}^{-1}$ and $1100 \mathrm{~cm}^{-1}$ caused by $\mathrm{Si}-$ OR stretching vibrations. These data suggested minimal oxidation. Furthermore, ${ }^{1} \mathrm{H}$ NMR spectra revealed a quarter peak between $3.6 \mathrm{ppm}$ and $3.75 \mathrm{ppm}$ (Fig. 1b), which we assigned to the proton adjacent to the amine group ${ }^{38}$. The peaks were not attributed to free allylamine, because the unreacted allylamine was removed via rotary evaporation. Our results indicate that the surface-modified Si-NPs were covered with allylamine and predominantly non-oxidized. The surface charge of the allylamine-modified Si-NPs was $+18.59 \mathrm{mV}$ (Supplementary Fig. S1).

Using transmission electron microscopy (TEM), we carried out additional observation of the allylamine-modified Si-NPs on a carboncoated copper grid. Monodispersed particles and atomic lattice planes were present (Fig. 1c), indicating a highly crystalline nature. The average diameter of 327 allylamine-modified Si-NPs was $\sim 3.4 \mathrm{~nm}$ (Fig. 1d), which is close to the Bohr radius of silicon $(4.3 \mathrm{~nm})^{39}$. The rings observed in the selected area electron diffraction pattern corresponded to crystalline silicon in the diamond structure (Fig. 1e). Energy-dispersive X-ray spectroscopy showed no platinum contamination (Supplementary Fig. S2), indicating that the catalyst was completely removed.

Next, we examined the optical character of allylamine-modified Si-NPs. Their absorption spectrum was wide (230-400 nm), with a shoulder peak at $300 \mathrm{~nm}$ (Fig. 1f) assigned to an $\Gamma-\Gamma$ band-gap trans- ition $^{38,40}$. The absorption maximum of $\sim 260 \mathrm{~nm}$ was indicative of the small size $(1-5 \mathrm{~nm})$ of the silicon nanocrystals ${ }^{41}$. The PL spectrum of allylamine-modified Si-NPs indicated peaks at $411 \mathrm{~nm}$ and $458 \mathrm{~nm}$ corresponding to excitation at $330 \mathrm{~nm}$ and $400 \mathrm{~nm}$, respectively (Fig. 1g). These data implied red shift in the PL peak position as the excitation wavelength increased. The blue luminescence assigned to the $\Gamma-\Gamma$ band-gap transition was in accordance with previous reports ${ }^{42,43}$. The time-resolved PL spectrum revealed that PL decay was rapid at $420 \mathrm{~nm}$, slightly slower at $440 \mathrm{~nm}$, and constant 460$520 \mathrm{~nm}$ (Fig. 1h). The time constants were $\sim 0.7 \mathrm{~ns}, 4 \mathrm{~ns}$, and $17 \mathrm{~ns}$, suggesting multiple de-excitation processes in allylamine-modified Si-NPs. The character of PL decay was consistent with direct bandgap recombination in $\mathrm{Si}-\mathrm{NPs}^{44}$.

Safety of allylamine-modified Si-NPs. To determine whether allylamine-modified Si-NPs are safe for human cells, we performed a water-soluble tetrazolium salt (WST) cell proliferation assay using 2 types of cells, 293XL-hTLR9 cells and peripheral blood mononuclear cells (PBMCs). The WST assay is based on the production of formazan from WST by mitochondrial dehydrogenases in viable cells. We detected no damage to either type of cell, even after exposure to a high dose of allylamine-modified Si-NPs for $24 \mathrm{~h}$ (Supplementary Fig. S3). When cells were exposed to $100 \mu \mathrm{g} / \mathrm{mL}$ of the surface-modified Si-NPs for $48 \mathrm{~h}$, the viability of the 293XLhTLR9 cells was reduced to $\sim 80 \%$ of that of the control cells, whereas no toxic effects on PBMCs were observed. Our results indicate that allylamine-modified Si-NPs are non-toxic to PBMCs.

Allylamine-modified Si-NPs as carriers for the delivery of CpG ODNs. TLR9 is localized in the endoplasmic reticulum and transferred to endolysosomes, where CpG ODNs interact with TLR9. Therefore, CpG ODNs must be delivered into endolysosomes. We investigated the uptake of allylamine-modified Si-NPs in 293XLTLR9 cells and observed that they were mainly localized in endolysosome-expressing, lysosome-associated membrane protein 1 (LAMP-1; Fig. 2a). This outcome attested to the suitability of allylamine-modified Si-NPs as carriers for the delivery of CpG ODNs to TLR9.

Next, we evaluated the maximum amount of CpG ODN2006x3PD bound electrostatically to allylamine-modified Si-NPs. CpG ODN2006x3-PD contains 72 bases and consists entirely of a phosphodiester backbone. When $40 \mu \mathrm{g}$ allylamine-modified Si-NPs was incubated with $46 \mu \mathrm{L} 300 \mathrm{ng} / \mu \mathrm{L}$ CpG ODN2006x3-PD solution at $4^{\circ} \mathrm{C}$ for $4 \mathrm{~h}, \sim 53 \%$ of the CpG ODN2006 3 -PD molecules were bound electrostatically to the surface of the Si-NPs. This result indicated that the maximum number of CpG ODN2006x3-PD molecules were bound to the allylamine-modified Si-NPs and that the surface had no further capacity for residual CpG ODNs binding. Therefore, the maximum amount of CpG ODN2006x3-PD loaded onto allylamine-modified Si-NPs was estimated to be $183 \mu \mathrm{g} / \mathrm{mg}$ NPs.

To verify the presence of CpG ODN molecules on the allylaminemodified Si-NPs, we mixed fluorescein isothiocyanate (FITC)labeled CpG ODN2006x3-PD molecules with allylamine-modified Si-NPs and observed the mixture using fluorescence microscopy. The emitted PL spectra from FITC and Si-NPs merged completely (Fig. 2b), confirming that CpG ODN2006x3-PD molecules were bound to the surface of the allylamine-modified Si-NPs. Furthermore, the thickness of the CpG ODN2006x3-PD layer on the allylamine-modified Si-NPs was $\sim 1 \mathrm{~nm}$ (Fig. $2 \mathrm{c}$ ). This observation suggested that CpG ODN2006x3-PD molecules had wound around the surface of the modified Si-NPs via electrostatic binding (Supplementary Fig. S4).

Cytokine induction by CpG ODNs electrostatically bound to allylamine-modified Si-NPs. Similar to class B CpG ODNs, free CpG ODN2006x3-PD molecules stimulated IL-6 production in PBMCs, including pDCs and B cells (Fig. 3a). However, CpG 


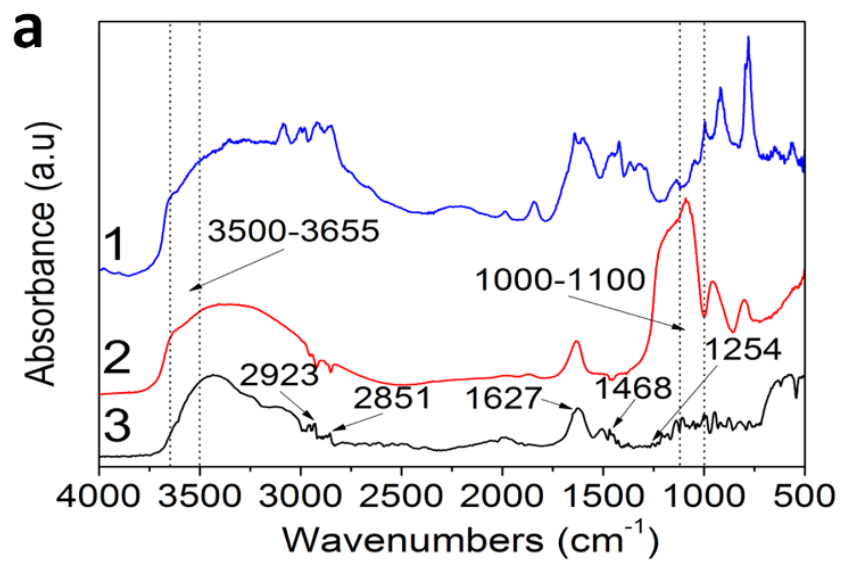

C

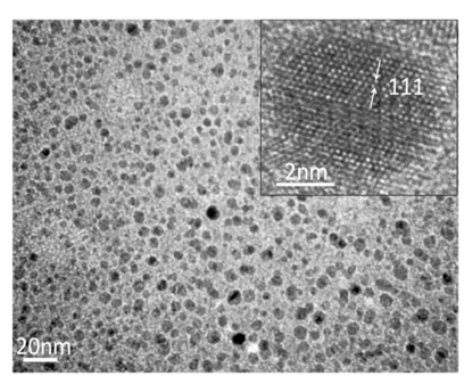

f

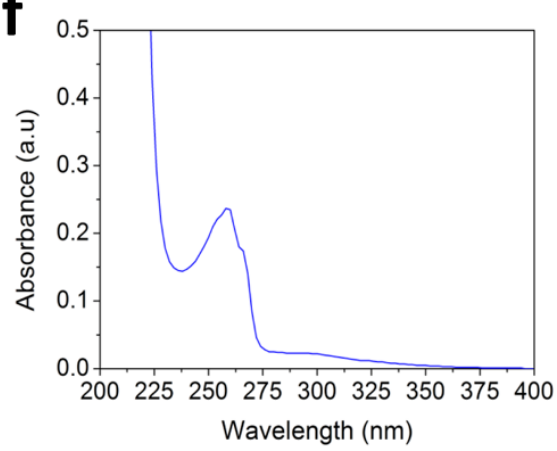

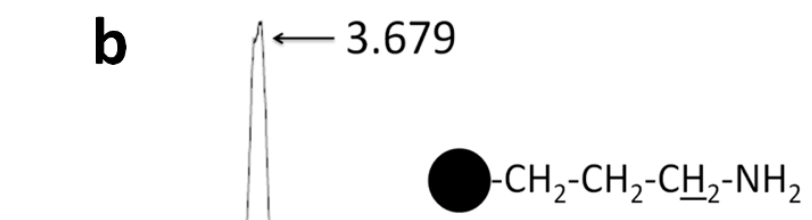

3.643

3.633
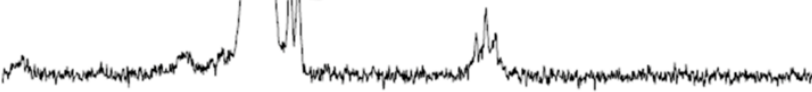

$\begin{array}{llllllllllll}3.9 & 3.8 & 3.7 & 3.6 & 3.5 & 3.4 & 3.3 & 3.2 & 3.1 & \mathrm{ppm}\end{array}$

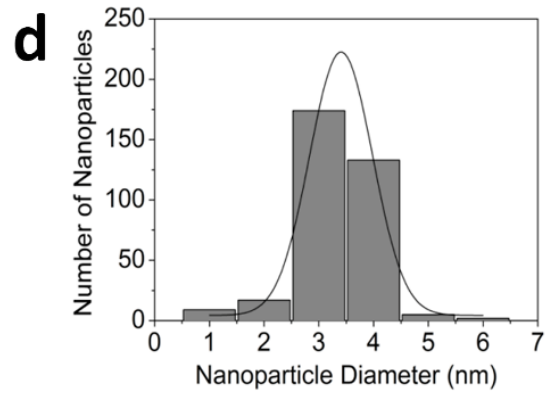

e

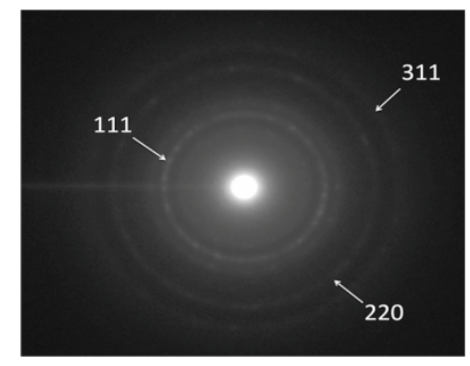

h
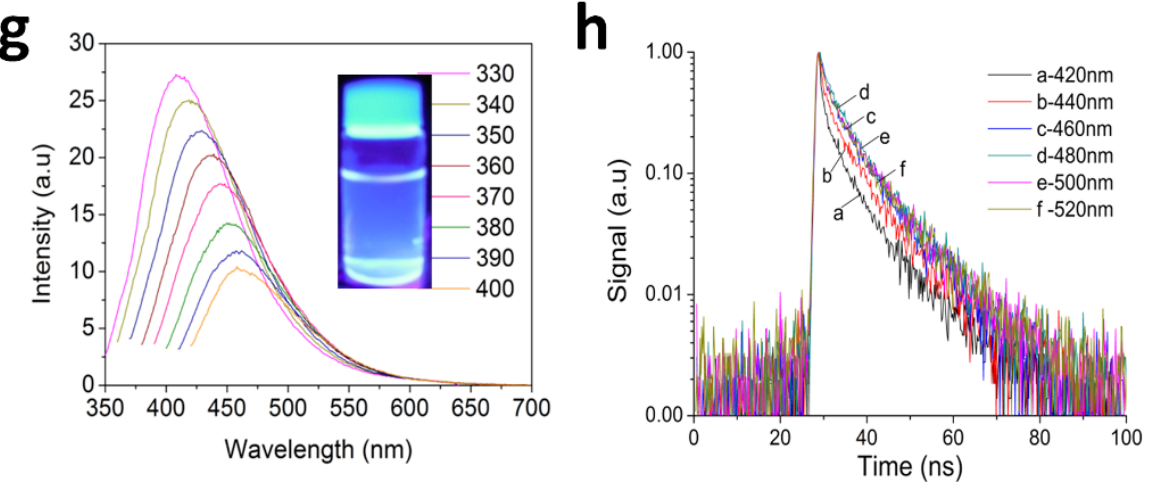

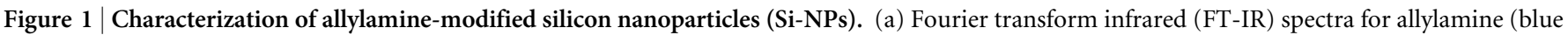

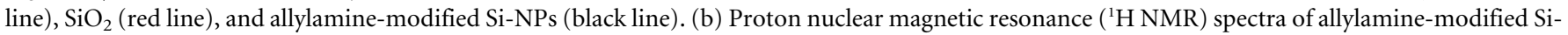
NPs in $\mathrm{CDCl}_{3}$. (c) Transmission electron microscopic (TEM) observation of allylamine-modified Si-NPs. The leaflet indicates crystal lattice planes.

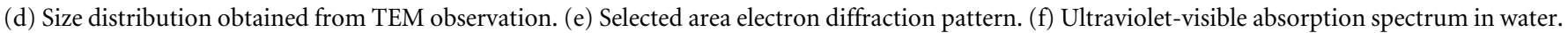

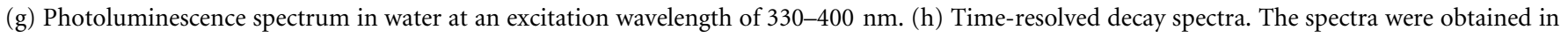
water using an emission wavelength range of 420-520 $\mathrm{nm}$ and an excitation wavelength of $400 \mathrm{~nm}$.

ODN2006x3-PD molecules loaded onto allylamine-modified Si-NPs suppressed IL- 6 production. By contrast, induction of IFN- $\alpha$ was observed in CpG ODN2006x3-PD molecules loaded onto allylamine-modified Si-NPs (Fig. 3b), whereas free CpG ODN2006x3$\mathrm{PD}$ molecules had no potential to stimulate IFN- $\alpha$ production.

We hypothesized that IFN- $\alpha$ production was stimulated by the CpG ODN2006x3-PD loaded onto the surface of allylamine-modified Si-NPs but not by free CpG ODN2006x3-PD molecules released from the Si-NPs. To verify this hypothesis, we examined whether CpG ODN2006x3-PD molecules were released from the surface of the Si-NPs. When FITC-labeled CpG ODN2006x3-PD molecules loaded onto allylamine-modified Si-NPs were added to the culture medium, the intracellular localization of CpG ODN2006x3-PD molecules corresponded strongly with that of the allylaminemodified Si-NPs (Fig. 4). This observation indicated that CpG ODN2006x3-PD molecules were not released from the allylaminemodified Si-NPs inside cells but remained on the Si-NP surface. To confirm this observation, we tested the level of free CpG
ODN2006x3-PD molecules released from the allylamine-modified Si-NPs in sodium acetate buffer ( $\mathrm{pH} \mathrm{5.0,} \mathrm{corresponding} \mathrm{to} \mathrm{that} \mathrm{in}$ endolysosomes). We detected no free CpG ODN2006x3-PD molecules (Supplementary Fig. S5), indicating that CpG ODN2006x3-PD molecules on the surface of allylamine-modified Si-NPs, but not free CpG ODN2006x3-PD molecules released from the Si-NPs, interact with TLR9, thereby stimulating IFN- $\alpha$ production.

Cytokine induction by CpG ODNs bound to Si-NPs through thiol-maleimide cross-linking. To examine the effect of binding mode of CpG ODNs on the surface of NPs, we introduced a maleimide group to the allylamine-modified Si-NPs using the allylamine amino group. We also prepared $3^{\prime}$-thiolated $\mathrm{CpG}$ ODN2006x3-PD molecules and cross-linked them on Si-NPs via a thiol-maleimide reaction (Supplementary Fig. S6). In this binding mode, CpG ODN2006x3-PD molecules are thought to grow from the Si-NP-like hairs, because only the $3^{\prime}$-end of CpG ODN2006x3-PD molecule binds to Si-NPs; the $5^{\prime}$-end is free (see Supplementary Fig. 
a

DIC

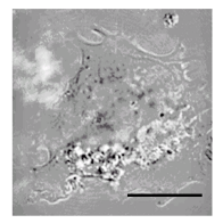

Si-NPs
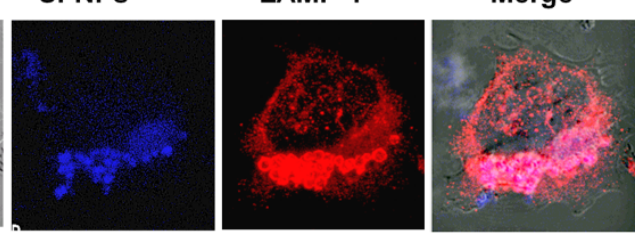

b

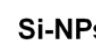
Si-NPs FITC-labeled ODN2006x3-PD Merge

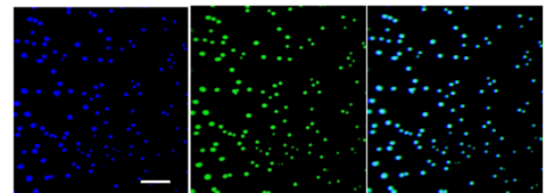

C

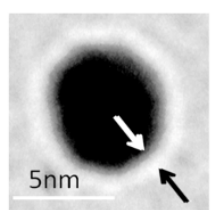

Figure $2 \mid$ Allylamine-modified Si-NPs as carriers of cytosine-phosphateguanine oligodeoxynucleotides (CpG ODNs). (a) Cellular localization of allylamine-modified Si-NPs. Immunostaining of the endolysosome marker, lysosome-associated membrane protein 1 (LAMP-1), is shown in red. Allylamine-modified Si-NPs are shown in blue and are localized in endolysosomes. DIC, differential interference contrast. Bar $=10 \mu \mathrm{m}$. (b) CpG ODN2006x3-PD molecules bound electrostatically to the surface of allylamine-modified Si-NPs. Allylamine-modified Si-NPs were mixed with fluorescein isothiocyanate (FITC)-labeled CpG ODN2006x3-PD and collected. Images were obtained with a fluorescence microscope. Bar $=5$ $\mu \mathrm{m}$. (c) TEM image of an allylamine-modified Si-NP loaded with CpG ODN2006x3-PD molecules. Arrows indicate the CpG ODN2006x3-PD layer. Bar $=5 \mathrm{~nm}$.

S6). When $40 \mu \mathrm{g}$ maleimide-modified Si-NPs was incubated with 46 $\mu \mathrm{L} 220 \mathrm{ng} / \mu \mathrm{L} 3^{\prime}$-thiolated CpG ODN2006x3-PD solution, $\sim 33 \%$ of the CpG ODN2006x3-PD molecules were cross-linked to the surface of the Si-NPs. Therefore, the maximum amount of $\mathrm{CpG}$ ODN2006x3-PD cross-linked to Si-NPs was estimated to be 72.6 $\mu \mathrm{g} / \mathrm{mg}$ NPs. CpG ODN2006x3-PD cross-linked to Si-NPs through the thiol-maleimide reaction induced a significantly higher level of IL-6 compared to that induced by free CpG ODN2006x3-PD with or without thiol modification (Fig. 5a). However, similar to free $\mathrm{CpG}$ ODN2006x3-PD, the cross-linked CpG ODN2006x3-PD was unable to stimulate IFN- $\alpha$ production (Fig. $5 \mathrm{~b}$ ). Thus, we propose that the binding mode of the CpG ODN2006x3-PD molecule affects cytokine induction.

Next, we investigated the intracellular localization of $\mathrm{CpG}$ ODN2006x3-PD molecules on Si-NPs. Free CpG ODN2006x3-PD molecules were localized in endolysosomes expressing LAMP-1 (Fig. 6a). CpG ODN2006x3-PD molecules cross-linked to maleimide-modified Si-NPs were localized in endolysosomes (Fig. 6b), but this localization was not observed in the molecules bound electrostatically to allylamine-modified Si-NPs (Fig. 6c).

Simultaneous induction of IFN- $\alpha$ and IL-6 using different binding modes of CpG ODNs on Si-NPs. To evaluate the effect of free CpG ODN2006x3-PD on IFN- $\alpha$ induction by CpG ODN2006x3$\mathrm{PD}$ bound electrostatically to allylamine-modified Si-NPs, we simultaneously added CpG ODN2006x3-PD molecules in both forms to the PBMC culture. Although IL-6 induction was observed, the level of IFN- $\alpha$ was significantly reduced compared to that resulting when $\mathrm{CpG}$ ODN2006x3-PD was loaded onto allylamine-modified $\mathrm{Si}-\mathrm{NPs}$ without free CpG ODN molecules (Fig. 7a, b). This result suggests that simultaneous induction of IFN- $\alpha$ and IL- 6 is difficult using both class A and class B CpG ODNs because free class B CpG ODN molecules that induce IL-6 inhibit IFN- $\alpha$ induction. However, we observed simultaneous induction of IFN- $\alpha$ and IL-6 when CpG ODN2006x3-PD
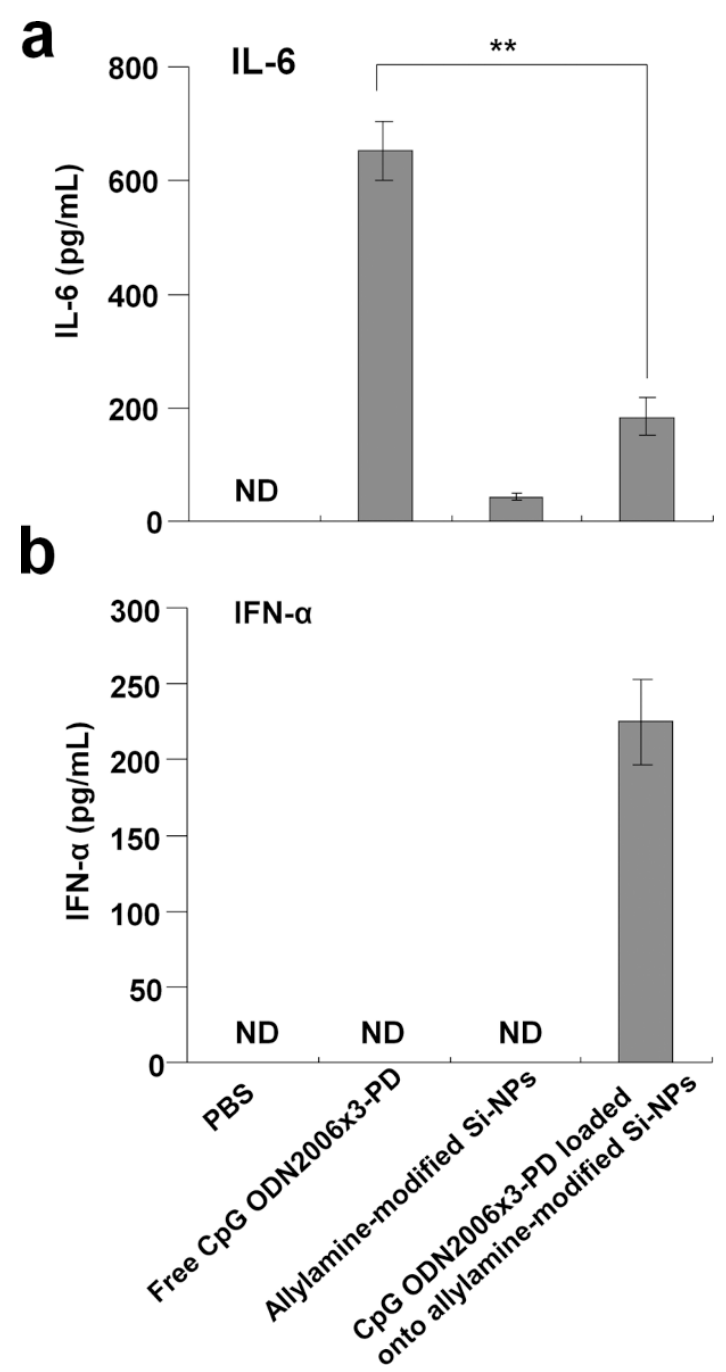

Figure $3 \mid$ Induction of interleukin-6 (IL-6) and interferon- $\alpha$ (IFN- $\alpha$ ) in peripheral blood mononuclear cells (PBMCs). Cells were stimulated with $0.65 \mu \mathrm{M}$ free CpG ODN2006x3-PD molecules or $80 \mu \mathrm{g} / \mathrm{mL}$ allylaminemodified Si-NPs loaded with CpG ODN2006x3-PD at a concentration corresponding to $0.65 \mu \mathrm{M}$ in the culture medium. (a) Free $\mathrm{CpG}$ ODN2006x3-PD molecules stimulated IL-6 induction, but CpG ODN2006x3-PD bound electrostatically to allylamine-modified Si-NPs inhibited the induction. $n=3$. ${ }^{* *}, p<0.05$. ND, not detected. (b) IFN- $\alpha$ was induced by CpG ODN2006x3-PD bound electrostatically to allylamine-modified Si-NPs but not by free CpG ODN2006x3-PD. $n=3$. ND, not detected; PBS, phosphate-buffered saline.

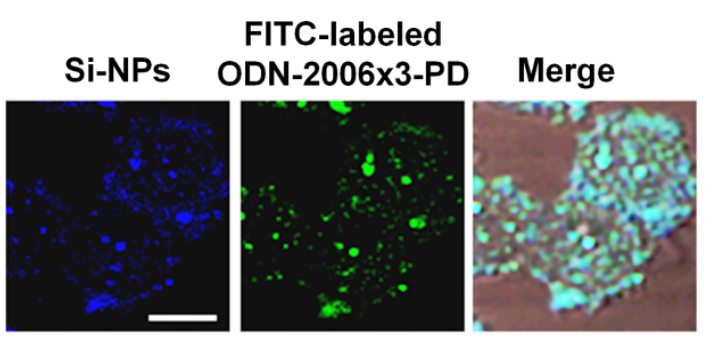

Figure $4 \mid$ Intracellular behavior of CpG ODN2006x3-PD. CpG ODN2006x3-PD molecules bound electrostatically to allylamine-modified Si-NPs remained in place inside cells. Bar $=10 \mu \mathrm{m}$. 
a

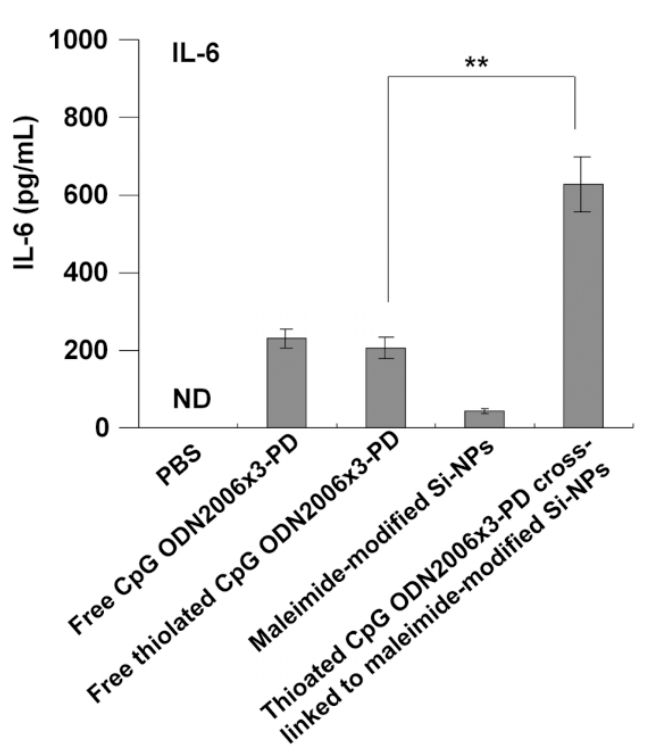

b

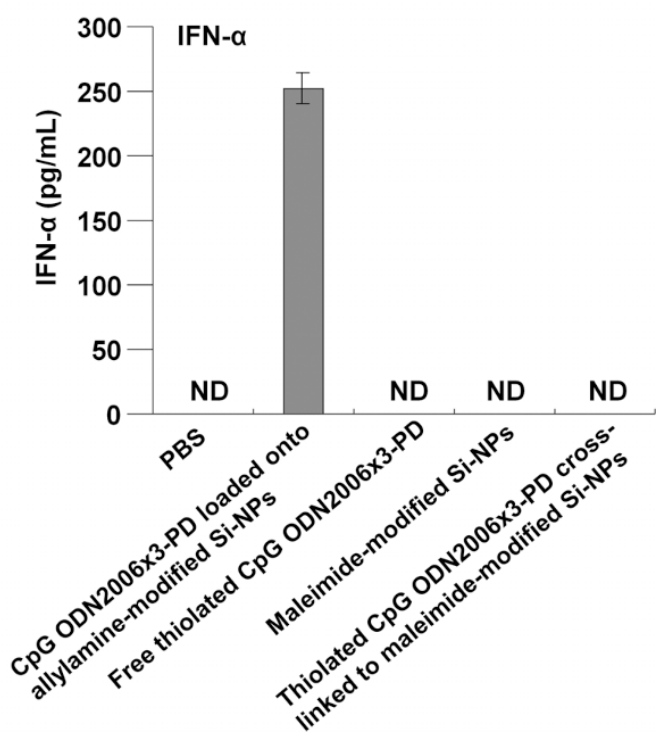

Figure 5 | Cytokine induction by thiolated CpG ODN2006x3-PD molecules cross-linked to maleimide-modified Si-NPs. (a) IL-6 induction. PBMCs were stimulated with $0.26 \mu \mathrm{M}$ free CpG ODN2006x3PD molecules and $80 \mu \mathrm{g} / \mathrm{mL}$ maleimide-modified Si-NPs loaded with thiolated CpG ODN2006x3-PD at a concentration corresponding to 0.26 $\mu \mathrm{M}$ in the culture medium. CpG ODN2006x3-PD molecules cross-linked to Si-NPs showed a higher potential to induce IL-6 than did free CpG ODNs. $n=3$. ${ }^{* *}, p<0.05$. (b) IFN- $\alpha$ induction. CpG ODN2006x3-PD molecules cross-linked to maleimide-modified Si-NPs showed no potential to induce IFN- $\alpha . n=3$.

molecules both bound electrostatically to allylamine-modified SiNPs and cross-linked to maleimide-modified Si-NPs were added to the PBMC culture (see Fig. 7a,b). This observation implies that, unlike free CpG ODN2006x3-PD molecules, CpG ODN2006×3PD molecules cross-linked to maleimide-modified Si-NPs with a potential to induce IL- 6 do not inhibit IFN- $\alpha$ induction.

\section{Discussion}

The difference in cytokine production between class A and class B $\mathrm{CpG}$ ODNs is thought to result from their sequence-dependent structures. Class A CpG ODN molecules reportedly form duplexes based on their palindromic sequences, and 2 duplexes further form a a

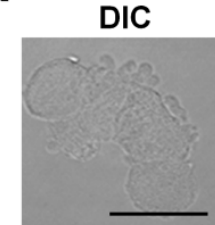

b

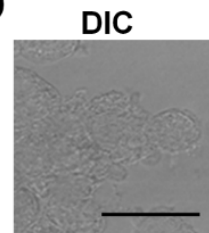

C
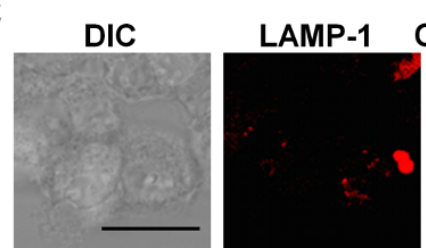

FITC-labeled LAMP-1 ODN2006x3-PD Merge
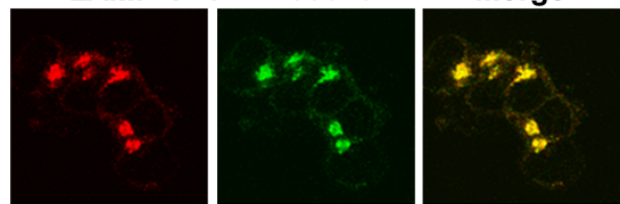

FITC-labeled

LAMP-1 ODN2006x3-PD
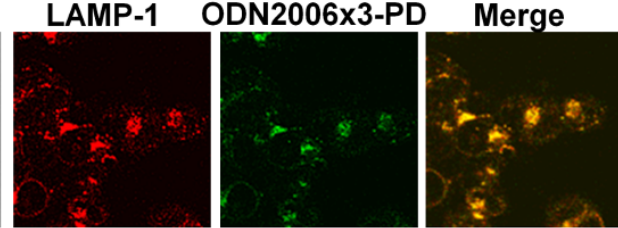

FITC-labeled

ODN2006x3-PD
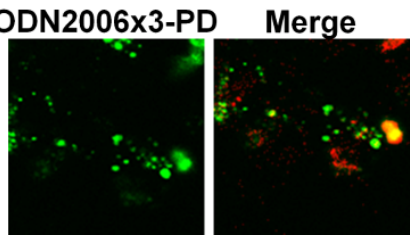

Figure $6 \mid$ Intracellular localization of CpG ODN2006x3-PD. (a) Free CpG ODN2006x3-PD was localized in endolysosomes expressing LAMP1. (b) CpG ODN2006x3-PD molecules cross-linked to maleimidemodified Si-NPs were localized in endolysosomes. (c) CpG ODN2006x3PD molecules bound electrostatically to allylamine-modified Si-NPs were not localized in endolysosomes. CpG ODN2006x3-PD molecules were labeled with FITC (green). Immunostaining of the endolysosome marker LAMP-1 is shown in red. DIC, differential interference contrast. Bar $=25$ $\mu \mathrm{m}$.

quadruplex because of the poly-G sequences at each $\mathrm{end}^{32,33}$. The quadruplex interacts with duplex or monomeric CpG ODN molecules, forming a higher-order structure. Class B CpG ODN molecules do not form such higher-order structures. Using atomic force microscopy (AFM), the size of the multimeric, higher-order structure was estimated to be $\sim 50 \mathrm{~nm}$ in diameter in CpG ODN2216 ${ }^{32}$, and $1.1 \pm 0.2 \mathrm{~nm}$ in height, $10.2 \pm 1.9 \mathrm{~nm}$ in width, and $30-70 \mathrm{~nm}$ in length in CpG ODN2336 ${ }^{33}$. CpG ODN2216 and ODN2336 are distinct class A CpG ODNs consisting of 20 and 21 bases, respectively. The class B CpG ODN, CpG ODN2007, consists of 22 bases and is a coil in solution; its size, estimated using AFM, is $1.2 \pm 0.3 \mathrm{~nm}$ in height and $8.7 \pm 1.4 \mathrm{~nm}$ in diameter ${ }^{33}$. Therefore, the class A CpG ODNs that interact with TLR9 are markedly larger than those in class B. This difference suggests that the size of CpG ODNs is critical for IFN- $\alpha$ induction. Moreover, class B CpG ODN molecules bound electrostatically to $180-\mathrm{nm}$ cationic polystyrene NPs induce IFN$\alpha^{32}$. We hypothesized that CpG ODNs larger than class A CpG ODNs have the potential to induce IFN- $\alpha$ regardless of class. Thus, we examined whether class B CpG ODN molecules loaded onto NPs smaller than multimeric class-A CpG ODNs could stimulate IFN- $\alpha$ induction.

For this purpose, we prepared allylamine-modified Si-NPs smaller than multimeric class A CpG ODNs. The average diameter of our allylamine-modified $\mathrm{Si}-\mathrm{NPs}$ was $3.4 \pm 0.2 \mathrm{~nm}$. Si-NPs with diameters of $<5 \mathrm{~nm}$ can be visualized as quantum dots (QDs), and this optical character can be applied in the field of bioimaging ${ }^{45-48}$. Using ultraviolet-visible (UV-Vis) absorption, we observed a maximum at $260 \mathrm{~nm}$, which was assigned to direct band-gap transition $^{49}$. Additionally, the maximum PL peak appeared at $411 \mathrm{~nm}$, with a full-width half-maximum height of $88 \mathrm{~nm}$ and an excitation wavelength at $330 \mathrm{~nm}$. The red shift in the emission wavelength may be attributed to excitation of the different sizes of Si-NPs ${ }^{50}$. 
a

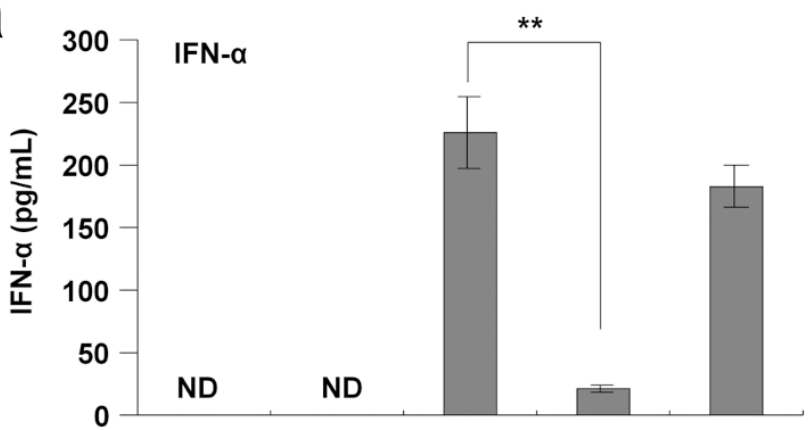

b

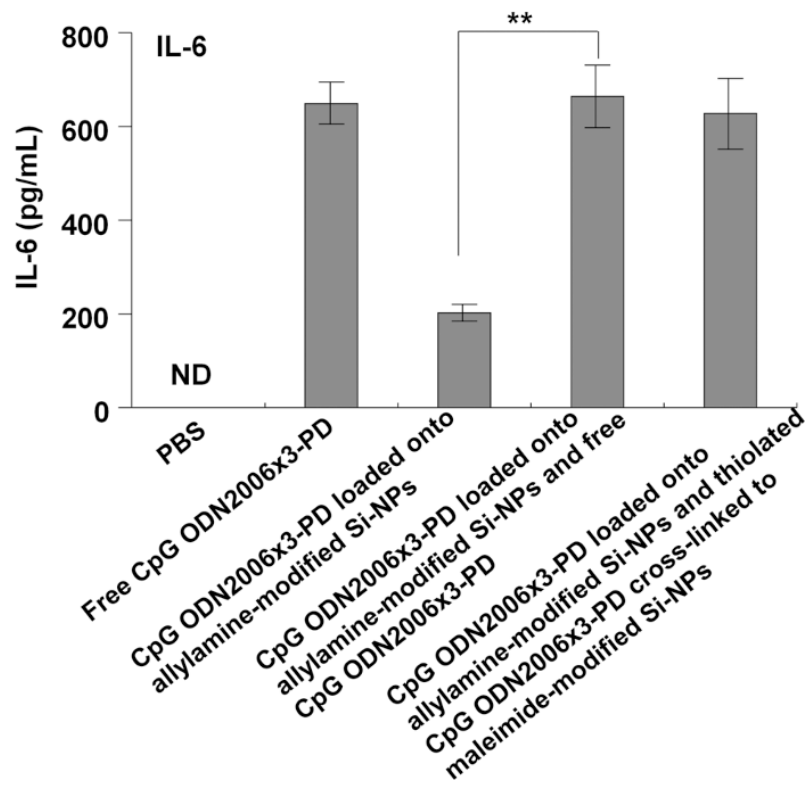

Figure 7 | Cytokine induction by CpG ODN2006x3-PD molecules bound electrostatically to allylamine-modified Si-NPs added to PBMCs with free CpG ODN2006x3-PD or CpG ODN2006x3-PD molecules cross-linked to maleimide-modified Si-NPs. (a) IFN- $\alpha$ induction was stimulated by 80 $\mu \mathrm{g} / \mathrm{mL}$ allylamine-modified Si-NPs loaded with CpG ODN2006x3-PD molecules but was strongly inhibited by the simultaneous addition of free CpG ODN2006x3-PD $(0.63 \mu \mathrm{M})$. Conversely, the inhibition was not observed in CpG ODN2006x3-PD molecules cross-linked to maleimidemodified Si-NPs. $n=3 .{ }^{* *}, p<0.05$. ND, not detected. (b) IL-6 induction was suppressed by CpG ODN2006x3-PD bound electrostatically to allylamine-modified Si-NPs, but CpG ODN2006x3-PD on allylaminemodified Si-NPs did not inhibit IL-6 induction by free CpG ODN2006x3PD or CpG ODN2006-PD cross-linked to maleimide-modified Si-NPs. $n=3 . * *, p<0.05$. ND, not detected.

Large band-gap transitions $(4.8 \mathrm{eV})$ are usually absorbed by $\mathrm{Si}$ nanocrystals with diameters of $<2 \mathrm{~nm}^{40}$. Cd-based QDs, such as $\mathrm{CdTe}, \mathrm{CdTe} / \mathrm{CdSe}$, and CdSe/ZnS, have been reported to show cytotoxicity $^{51-54}$. However, our allylamine-modified Si-NP showed no toxicity to 293XL-hTLR9 cells and PBMCs, even at concentrations of $80 \mu \mathrm{g} / \mathrm{mL}$ and $100 \mu \mathrm{g} / \mathrm{mL}$, respectively. The cytotoxicity of QDsized NPs may affect cytokine induction by immune cells and therefore the non-toxic allylamine-modified Si-NPs were useful for the present study. Allylamine-modified Si-NPs were taken up by cells and mainly localized in endolysosomes expressing LAMP-1. This localization is advantageous for CpG ODN delivery, because TLR9 is also localized in endolysosomes. Allylamine-modified Si-NPs have a positive charge and robust dispersibility, and therefore, CpG ODN molecules were easily bound to the Si-NPs. Additionally, we were able to analyze the intracellular behavior of CpG ODN molecules and Si-NPs using the PL emitted from FITC and Si-NPs when FITC-labeled CpG ODN molecules were loaded onto the $\mathrm{Si}-\mathrm{NPs}$ as QDs.

The CpG ODN2006x3-PD molecules used in the present study consisted of 72 bases, and were $>3$ times longer than class B CpG ODN2007 molecules (22 bases). The distance between bases in a double-stranded helix structure is $0.34 \mathrm{~nm}(3.4 \AA)$, and therefore the length of the monomeric single-stranded CpG ODN2007 is believed to be $>7.14 \mathrm{~nm}(0.34 \mathrm{~nm} \times 21$ bases $)$. This length corresponds closely with the size estimated using means of AFM $(8.7 \pm 1.4 \mathrm{~nm})^{33}$. The length of the CpG ODN2006x3-PD molecule is estimated to be $>24 \mathrm{~nm}(0.34 \mathrm{~nm} \times 71$ bases), which is comparable to the minimum size of class A CpG ODNs with multimeric higher-order structures $(30 \mathrm{~nm})^{33}$. However, no IFN- $\alpha$ induction was observed in free CpG ODN2006x3-PD molecules, indicating that larger class B CpG ODN molecules lacks the potential to stimulate IFN- $\alpha$ induction. By contrast, CpG ODN2006x3-PD molecules bound electrostatically to allylamine-modified Si-NPs stimulated IFN- $\alpha$ induction. The average size of the NPs after electrostatic binding of CpG ODN2006x3-PD molecules was estimated to be $\sim 5.4 \mathrm{~nm}$ because the average diameter of allylamine-modified Si-NPs was $3.4 \mathrm{~nm}$ and the thickness of the CpG ODN2006x3-PD layer on the $\mathrm{Si}$-NPs was $\sim 1 \mathrm{~nm}$. NPs loaded with CpG ODN molecules are much smaller than multimeric class A CpG ODNs. However, class B CpG ODN2006x3-PD molecules bound electrostatically to Si-NPs smaller than multimeric class A CpG ODNs with higher-order structures also have the potential to induce IFN- $\alpha$. Our data suggest that the increased size of class A CpG ODNs resulting from self-assembly does not contribute to IFN- $\alpha$ induction.

The thickness of the CpG ODN2006x3-PD layer on the allylamine-modified $\mathrm{Si}-\mathrm{NPs}$ was $\sim 1 \mathrm{~nm}$, which is much shorter than the length of the monomeric CpG ODN2006x3-PD molecule. This result implies that many $\mathrm{CpG}$ ODN2006x3-PD molecules are randomly wound around the Si-NP and form a compacted 1-nm layer. That is, the whole molecule of CpG ODN2006x3-PD is entirely bound to the surface of allylamine-modified Si-NPs, because every negatively charged phosphate group of the nucleotides that constitute the CPG ODN molecule is involved in electrostatic binding to the positively charged allylamine amino groups. PL emitted from FITC and Si-NPs revealed that CpG ODN2006x3-PD molecules were not released from Si-NPs inside cells. Furthermore, CpG ODN2006x3-PD molecules released from Si-NPs were not detected in sodium acetate buffer, which has a $\mathrm{pH}$ similar to that of endolysosomes. These observations imply that multiple molecules of $\mathrm{CpG}$ ODN2006x3-PD on Si-NPs, but not monomers released from SiNPs, interact with TLR9, thereby leading to IFN- $\alpha$ production. We further observed that free CpG ODN2006x3-PD molecules inhibited IFN- $\alpha$ production by CpG ODN2006x3-PD bound electrostatically to Si-NP, which also indicates that only CpG ODN2006x3PD molecules on Si-NPs are involved in IFN- $\alpha$ induction. The size, chemical composition, and surface modification of NPs are thought to be uninvolved in the stimulation of IFN- $\alpha$ induction, because class B CpG ODNs loaded onto $180-\mathrm{nm}$ cationic polystyrene NPs also induce IFN- $\alpha^{32}$. Therefore, multimerization of $\mathrm{CpG}$ ODN molecules via self-assembly or using NPs is a critical factor for of IFN- $\alpha$ induction.

To investigate further the effects of the multimerization of $\mathrm{CpG}$ ODN2006x3-PD molecules, we prepared an alternate binding mode using covalent-cross-linking between 3 '-thiolated CpG ODN molecules and maleimide that was introduced on the Si-NP surface. This binding mode induced IL- 6 but not IFN- $\alpha$, as did free CpG ODN2006x3-PD. In this binding mode, only the 3 '-end of CpG ODN2006x3-PD molecules is bound to the Si-NP, and the $5^{\prime}$-end, which has a binding mode just like hair, is free. Thus, we believe that CpG ODN molecules bound to the surface of Si-NPs through covalent cross-linking may behave in the same manner observed for those in the monomeric form. These results indicate that multimerization 

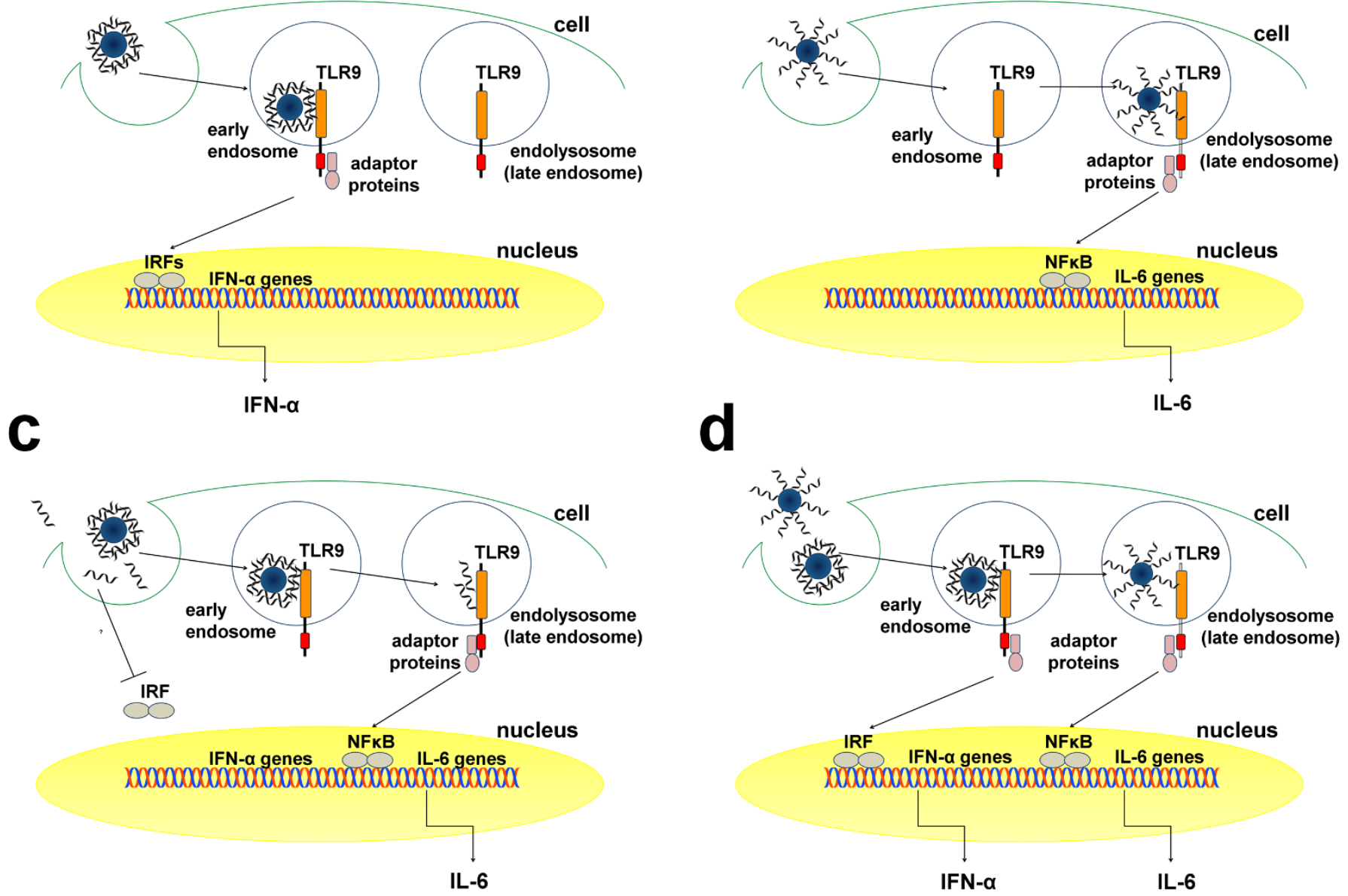

Figure 8 Proposed model for binding mode-dependent bifurcation of cytokine induction in class B CpG ODN2006x3-PD. (a) CpG ODN2006x3-PD molecules bound electrostatically onto allylamine-modified Si-NPs are mainly localized in early endosomes and induce IFN- $\alpha$, although free CpG ODN2006x3-PD molecules are localized in endolysosomes (late endosomes) and induce only IL-6. IRFs, interferon regulatory factors. (b) CpG ODN2006x3-PD molecules cross-linked to maleimide-modified Si-NPs are localized in endolysosomes and induce IL-6 but not IFN- $\alpha$. (c)Free CpG ODN2006x3-PD molecules inhibit IFN- $\alpha$ induction by CpG ODN2006x3-PD bound electrostatically onto allylamine-modified Si-NPs. However, the inhibition mechanism remains unclear. (d) CpG ODN2006x3-PD molecules cross-linked to maleimide-modified Si-NPs do not inhibit IFN- $\alpha$ induction by CpG ODN2006x3-PD bound electrostatically onto allylamine-modified Si-NPs, thereby stimulating both IFN- $\alpha$ and IL-6.

of CpG ODN molecules is required for IFN- $\alpha$ induction, but the method of multimerization significantly affects cytokine induction.

We observed that CpG ODN2006x3-PD molecules cross-linked onto maleimide-modified Si-NPs were localized in endolysosomes expressing LAMP-1, whereas the CpG ODN molecules electrostatically bound to allylamine-modified Si-NPs were not localized in the endolysosomes. Free CpG ODN2006x3-PD molecules were localized in the endolysosomes, as were the CpG ODN molecules cross-linked to maleimide-modified Si-NPs. These observations suggest that the binding mode, but not the presence or absence of NP, affects the localization of CpG ODNs. Monomeric class B CpG ODN molecules are reportedly translocated to late endosomes, leading to the induction of proinflammatory cytokines such as IL-6, whereas multimeric class A CpG ODNs are retained in early endosomes, leading to IFN induction ${ }^{55-58}$. In addition, retention of class B CpG ODN molecules in early endosomes by means of complex formation with a cationic lipid or multimerization by $3^{\prime}$ poly-G extension is associated with IFN induction ${ }^{57,58}$. Therefore, we believe that the difference in binding mode of CpG ODN2006x3-PD molecules to Si-NPs affects the localization of $\mathrm{CpG}$ ODNs, resulting in different cytokine induction.

Free CpG ODN2006x3-PD molecules inhibited IFN- $\alpha$ induction by CpG ODN2006x3-PD bound electrostatically to Si-NPs.
Monomeric class B CpG ODNs reportedly inhibit class A CpG ODN-induced association of IFN regulatory factor 1 with promoters of IFNs, resulting in the inhibition of IFN induction ${ }^{59}$. By contrast, CpG ODN2006x3-PD molecules cross-linked to maleimide-modified Si-NPs showed no inhibitory effect on IFN- $\alpha$ induction by CpG ODN2006x3-PD molecules bound electrostatically to Si-NPs. This result implies that both IL- 6 and IFN- $\alpha$ can be induced simultaneously using different binding modes of class B CpG ODNs on NPs, although inducing both cytokines simultaneously by combining multimeric class A CpG ODNs and monomeric class B CpG ODNs or combining class-B CpG ODNs bound electrostatically to NPs and free class-B CpG ODNs is impossible.

We summarized our present findings in Fig. 8. This study demonstrated that the multimerization of class B CpG ODNs is required for IFN- $\alpha$ induction, but the binding mode of class B CpG ODNs to NPs significantly affects IFN- $\alpha$ induction when using NPs.

\section{Methods}

Synthesis of allylamine-modified Si-NPs. Allylamine-modified Si-NPs were prepared according to the procedure of Warner et al..$^{60}$, with minor modifications. Briefly, $92 \mu \mathrm{L} \mathrm{SiCl}_{4}(0.8 \mathrm{mM})$ was added to $100 \mathrm{~mL}$ anhydrous toluene containing $1.5 \mathrm{~g}$ tetraoctyl ammonium bromide and mixed with a homogenizer for $15 \mathrm{~min}$. Excess reducing agent $\left(\mathrm{LiAlH}_{4}\right)$ was reacted with the mixture for $3 \mathrm{~h}$, after which 
$20 \mathrm{~mL}$ methanol was slowly added to remove excess $\mathrm{LiAlH}_{4}$. Next, $92 \mu \mathrm{L} 0.1 \mathrm{M}$ chloroplatinic acid in isopropyl alcohol was added to hydrogen-terminated Si-NPs. To positively charge the surface, we added $5 \mathrm{~mL}$ allylamine to the mixture, and the solvent was removed via rotary evaporation at $65^{\circ} \mathrm{C}$ followed by vacuum drying for $2 \mathrm{~h}$. The powder of allylamine-modified Si-NPs was dispersed in $50 \mathrm{~mL}$ of Milli-Q water, and contaminated tetraoctyl ammonium bromide was removed via filtration using a $0.22-\mu \mathrm{m}$ polyvinylidene difluoride filter. For further purification, the Si-NPs were collected using a macrospin column G-25 (Harvard Apparatus, MA, USA). The allylamine-modified Si-NPs were dispersed in Milli-Q water at a concentration of $1 \mathrm{mg} / \mathrm{mL}$ and stored at room temperature without light. All of the chemicals were handled either in a glove box or on a Schlenk line under a nitrogen atmosphere.

Characterization of allylamine-modified Si-NPs. To confirm the surface modification of Si-NPs with allylamine, we used FT-IR (Spectrum GX, Perkin Elmer, USA) and ${ }^{1} \mathrm{H}$ NMR (DRX-600, Bruker, Germany) spectroscopy. FT-IR spectra were obtained at $4 \mathrm{~cm}^{-1}$ resolution with 32 scans. For ${ }^{1} \mathrm{H}$ NMR, Si-NPs were vacuum-dried and dissolved in $\mathrm{CDCl}_{3}$.

High-resolution TEM images were obtained with a JEOL3000F (Tokyo, Japan) using a copper grid with a thin carbon film and an acceleration voltage of $200 \mathrm{kV}$. $\mathrm{UV}$-vis absorption spectra were acquired on an UV-vis spectrophotometer (Hitachi U-2900, Japan). Fluorescence spectra were measured with a fluorescence spectrometer (Hitachi F-7000, Japan). The excitation and emission slit widths were $10 \mathrm{~nm}$.

The surface zeta potential of Si-NPs was measured at a concentration of $1.0 \% \mathrm{w} / \mathrm{v}$ in water using a laser electrophoresis zeta-potential analyzer (LEZA-600, Otsuka, Japan). Time-resolved decay spectra were obtained using a Spectra Pro-300-I (Acton Research Corporation, USA) spectrometer, a streak camera (C5860, Hamamatsu Photonics K.K., Japan) with a 400-nm excitation wavelength, and $500-n s$ pulses sourced from a frequency-doubled, mode-locked Ti: sapphire laser (Mira 900-F, Coherent, USA)

Cell cultures. PMBCs and 293XL-hTLR9 cells were purchased from Invitrogen (CA USA) and Cellular Technology (OH, USA), respectively. The 293XL-hTLR9 cells were grown in high-glucose, Dulbecco-modified Eagle's medium supplemented with $10 \%$ fetal bovine serum, 50 units $/ \mathrm{mL}$ penicillin, $50 \mathrm{mg} / \mathrm{mL}$ streptomycin, and $10 \mu \mathrm{g} / \mathrm{mL}$ blasticidin, at $37^{\circ} \mathrm{C}$ in humidified air containing $5 \% \mathrm{CO}_{2}$. The PBMCs were cultured according to manufacturer instructions.

Cytotoxic assay. An in vitro cytotoxic assay for allylamine-modified Si-NPs was performed using a Cell Counting Kit-8 (Dojindo, Japan). The cells were seeded in a 96 -well plate at a density of 5000 cells/well. After $24 \mathrm{~h}$ and $48 \mathrm{~h}$, allylamine-modified Si-NPs were added, and the culture plate was incubated for an additional $24 \mathrm{~h}$. After exposure, the cells were incubated with $10 \mu \mathrm{L}$ cholecystokinin octapeptide for $2 \mathrm{~h}$, and the absorbance at $450 \mathrm{~nm}$ was measured using a microplate reader (MTP$880 \mathrm{Lab}$, Corona, Japan). The cytotoxicity was represented as a percentage against untreated control cells.

Preparation of Si-NPs loaded with CpG ODN2006x3-PD. The sequence of the CpG ODN2006x3-PD molecule was 5'-TCGTCGTTTTGTCGTTTTGTCGTTTCGTCGTTTTGTCGTTTTGTCGTTTCGTCGTTTTGTCGTTTTGTCGTT-3' (72 bases), and it consisted entirely of a phosphodiester backbone ${ }^{31}$. CpG ODN2006x3-PD was dissolved in Milli-Q water at a concentration of $100 \mu \mathrm{M}$ and stored at $-20^{\circ} \mathrm{C}$ until use.

To study electrostatic binding of CpG ODN2006x3-PD to Si-NPs, we added $6 \mu \mathrm{L}$ CpG ODN2006x3-PD stock solution to $40 \mu \mathrm{L}$ allylamine-modified Si-NP stock solution to a final CpG ODN2006x3-PD concentration of $300 \mathrm{ng} / \mathrm{mL}$. The mixture was shaken using a rotary shaker at $4{ }^{\circ} \mathrm{C}$ for $4 \mathrm{~h}$ and then centrifuged at $150,000 \times g$ for $20 \mathrm{~min}$. The pellet of allylamine-modified Si-NPs loaded with CpG ODN2006x3PD molecules was resuspended with $46 \mu \mathrm{L}$ phosphate-buffered saline (PBS) buffer. The binding capacity of CpG ODN2006x3-PD loaded onto allylamine-modified SiNPs was estimated by determining the residual amount of CpG ODN2006x3-PD molecules in the supernatant using a NanoDrop spectrophotometer (Thermo Scientific, USA)

To explore the alternate binding mode of CpG ODN2006x3-PD molecules to Si$\mathrm{NPs}$, we reacted $1 \mathrm{mg} / \mathrm{mL}$ allylamine-modified Si-NPs with $100 \mu \mathrm{L}$ 4- $(\mathrm{N}$-maleimidomethyl)cyclohexane-1-carboxylic acid $N$-hydroxysuccinimide ester solution for $1 \mathrm{~h}$, and the mixture was centrifuged at $150,000 \times g$ for $20 \mathrm{~min}$ to remove unreacted 4-( $N$-maleimidomethyl)cyclohexane-1-carboxylic acid $N$-hydroxysuccinimide ester. The resulting pellet contained the maleimide-modified Si-NPs. To cross-link CpG ODN2006x3-PD molecules to maleimide-modified Si-NPs, we added $6 \mu \mathrm{L} 3^{\prime}$-thiolmodified CpG ODN2006x3-PD molecules to $40 \mu \mathrm{L}$ maleimide-modified Si-NP suspension to a final $3^{\prime}$-thiol-modified CpG ODN2006x3-PD concentration of $220 \mathrm{ng} / \mu \mathrm{L}$. The mixture was shaken with a rotary shaker at $4{ }^{\circ} \mathrm{C}$ for $4 \mathrm{~h}$ and centrifuged at $150,000 \times g$ for $20 \mathrm{~min}$. The pellet of maleimide-modified Si-NPs loaded with $3^{\prime}$-thiol-modified CpG ODN2006x3-PD was re-suspended with $46 \mu \mathrm{L}$ PBS buffer. The binding capacity of $3^{\prime}$-thiolated CpG ODN2006x3-PD on maleimidemodified Si-NPs was estimated by determining the residual amount of 3 '-thiolated CpG ODN2006x3-PD molecules in the supernatant using a NanoDrop spectrophotometer (Thermo Scientific).

Fluorescence microscopy. To analyze the intracellular localization of CpG ODN, we labeled the 3 '-end of CpG ODN2006x3-PD molecules with FITC. Allylaminemodified Si-NPs with or without FITC-labeled CpG ODN2006x3-PD were added at a final concentration of $80 \mu \mathrm{g} / \mathrm{mL}$ to 293XL-hTLR9 cells cultured in a 35-mm dish for $24 \mathrm{~h}$. The cells were then washed 3 times with PBS and fixed with $3.7 \%$ formaldehyde for $20 \mathrm{~min}$. Endolysosomes were stained with anti-LAMP-1 antibody (Abcam, Cambridge, UK). The differential interference contrast and fluorescence images were obtained with a confocal laser scanning fluorescence microscope (SP5, Leica Microsystems, Germany).

Cytokine assay. PBMCs were seeded in a 96-well plate at a density of $1 \times 10^{6}$ cells/well. The cells were immediately stimulated with CpG ODNs. After $48 \mathrm{~h}$, the supernatant was collected and assayed for IL- 6 using Ready-Set-Go! (eBiosciences, CA, USA) for enzyme-linked immunosorbent assay. For the IFN- $\alpha$ assay, we used a Human IFNModule Set enzyme-linked immunosorbent assay (eBiosciences).

Statistical analysis. Statistical significance was evaluated using a two-tailed, unpaired Student's $t$-test. A $p$ value of $<0.05$ was accepted as significant.

1. Hemmi, H. et al. A Toll-like receptor recognizes bacterial DNA. Nature 408, 740745 (2000).

2. Takeshita, F. et al. Cutting edge: Role of Toll-like receptor 9 in CpG DNA-induced activation of human cells. J. Immunol. 167, 3555-3558 (2001).

3. Krieg, A. M. CpG motifs in bacterial DNA and their immune effects. Annu. Rev. Immunol. 20, 709-760 (2002).

4. Hornung, V. et al. Quantitative expression of toll-like receptor 1-10 mRNA in cellular subsets of human peripheral blood mononuclear cells and sensitivity to CpG oligodeoxynucleotides. J. Immunol. 168, 4531-4537 (2002).

5. Krieg, A. M. et al. CpG motifs in bacterial DNA trigger direct B-cell activation. Nature 374, 546-549 (1995).

6. Klinman, D. M., Yi, A. K., Beaucage, S. L., Conover, J. \& Krieg, A. M. CpG motifs present in bacteria DNA rapidly induce lymphocytes to secrete interleukin 6, interleukin 12, and interferon $\gamma$. Proc. Natl. Acad. Sci. U.S.A. 93, 2879-2883 (1996).

7. Akira, S., Uematsu, S. \& Takeuchi, O. Pathogen recognition and innate immunity. Cell 124, 783-801 (2006).

8. Asselin-Paturel, C. et al. Type I interferon dependence of plasmacytoid dendritic cell activation and migration. J. Exp. Med. 201, 1157-1161 (2005).

9. Napolitani, G., Rinaldi, A., Bertoni, F., Sallusto, F. \& Lanzavecchia, A. Selected Toll-like receptor agonist combinations synergistically trigger a $\mathrm{T}$ helper type 1 polarizing program in dendritic cells. Nat. Immunol. 6, 769-776 (2005).

10. Roman, M. et al. Immunostimulatory DNA sequences function as T helper-1promoting adjuvants. Nat. Med. 3, 849-854 (1997).

11. Hartmann, G., Weiner, G. J. \& Krieg, A. M. CpG DNA: a potent signal for growth activation, and maturation of human dendritic cells. Proc. Natl. Acad. Sci. U.S.A. 96, 9305-9319 (1999).

12. Blackwell, S. E. \& Krieg, A. M. CpG-A-induced monocyte IFN-gamma-inducible protein-10 production is regulated by plasmacytoid dendritic cell-derived IFNalpha. J. Immunol. 170, 4061-4068 (2003).

13. Vollmer, J. et al. CpG oligodeoxynucleotides stimulate IFN-gamma-inducible protein-10 production in human B cells. J. Endotoxin. Res. 10, 431-438 (2004).

14. Krieg, A. M. Therapeutic potential of Toll-like receptor 9 activation. Nat. Rev. 5 , 471-484 (2006)

15. Jahrsdörfer, B. \& Weiner, G. J. CpG oligodeoxynucleotides for immune stimulation in cancer immunotherapy. Curr. Opin. Investig. Drugs 4, 686-690 (2003).

16. Fonseca, D. E. \& Kline, J. N. Use of CpG oligonucleotides in treatment of asthma and allergic disease. Adv. Drug Deliv. Rev. 61, 256-262 (2009).

17. Klinman, D. M., Klaschik, S., Sato, T. \& Tross, D. CpG oligonucleotides as adjuvants for vaccines targeting infectious diseases. Adv. Drug Deliv. Rev. 61, 248255 (2009).

18. Krug, A. et al. Identification of CpG oligonucleotide sequences with high induction of IFN-alpha/beta in plasmacytoid dendritic cells. Eur. J. Immunol. 31, 2154-2163 (2001).

19. Gürsel, M., Verthelyi, D., Gürsel, I., Ishii, K. J. \& Klinman, D. M. Differential and competitive activation of human immune cells by distinct classes of $\mathrm{CpG}$ oligonucleotide. J. Leukocyte Biol. 71, 813-820 (2002).

20. Hartmann, G. et al. Delineation of a CpG phosphorothioate oligodeoxynucleotide for activating primate immune responses in vitro and in vivo. J. Immunol. 164, 1617-1624 (2000).

21. Hartmann, G. \& Krieg, A. M. Mechanism and function of a newly identified CpG DNA motif in human primary B cells. J. Immunol. 164, 944-953 (2000).

22. Kurreck, J. Antisense technologies. Improvement through novel chemical modifications. Eur. J. Biochem. 270, 1628-1644 (2003).

23. Agrawal, S. \& Zhao, Q. Antisense therapeutics. Curr. Opin. Chem. Biol. 2, 519-528 (1998).

24. Mutwiri, G. K., Nichani, A. K., Babiuk, S. \& Babiuk, L. A. Strategies for enhancing the immunostimulatory effects of CpG oligodeoxynucleotides. J. Control Release 97, 1-17(2004).

25. Sheehan, J. P. \& Lan, H-C. Phosphorothioate oligonucleotides inhibit the intrinsic tenase complex. Blood 92, 1617-1625 (1998).

26. Brown, D. A. et al. Effect of phosphorothioate modification of oligodeoxynucleotides on specific protein binding. J. Biol. Chem. 269, 26801-26805 (1994). 
27. Levin, A. A. A review of the issues in the pharmacokinetics and toxicology of phosphorothioate antisense of oligonucleotides. Biochim. Biophys. Acta 1489, 6984 (1999).

28. Henry, S. P. et al. Complement activation is responsible for acute toxicities in rhesus monkeys treated with a phosphorothioate oligonucleotide. Int. Immunopharmacol. 2, 1657-1666 (2002).

29. Chavany, C., Connolly, Y. \& Neckers, L. Contribution of sequence and phosphorothioate content to inhibition of cell growth and adhesion caused by cmyc antisense oligomers. Mol. Pharmacol. 48, 738-746 (1995).

30. Crooke, R. M. In vitro toxicology and pharmacokinetics of antisense oligonucleotides. Anticancer Drug Des. 6, 609-646 (1991).

31. Meng, W., Yamazaki, T., Nishida, Y. \& Hanagata, N. Nuclease-resistant immunostimulatory phosphodiester CpG oligodeoxynucleotides as human Tolllike receptor 9 agonists. BMC Biotechnol. 11, 88-96 (2011).

32. Kerkmann, M. et al. Spontaneous formation of nucleic acid-based nanoparticles is responsible for high interferon- $\alpha$ induction by $\mathrm{CpG}-\mathrm{A}$ in plasmacytoid dendritic cells. J. Biol. Chem. 280, 8086-8093 (2005).

33. Klein, D. C. G., Latz, E., Espevik, T. \& Stokke, B. T. Higher order structure of short immunostimulatory oligonucleotides studied by atomic force microscopy. Ultramicroscopy 110, 689-693 (2010).

34. Mayne, A. H., Bayliss, S. C., Barr, P., Tobin, M. \& Buckbery, L. D. Biologically interfaced porous silicon devices. Phys. Status Solidi. A 182, 505-513 (2000).

35. Meyer, O. et al. Cationic liposomes coated with polyethylene glycol as carriers for oligonucleotides. J. Biol. Chem. 273, 15621-15627 (1998).

36. Mahato, R. I. et al. Biodistribution and expression of lipid/plasmid complexes after systemic administration. Hum. Gene Ther. 9, 2083-2099 (1998).

37. Kim, H., Akagi, T. \& Akashi, M. Preparation of CpG ODN-encapsulated anionic poly (amino acid) nanoparticles for gene delivery. Chem. Lett. 39, 278-279 (2010).

38. Shiohara, A. et al. Chemical reactions on surface molecules attached to silicon quantum dots. J. Am. Chem. Soc. 132, 248-253 (2010).

39. Fujioka, K. et al. Luminescent passive-oxidized silicon quantum dots as biological staining labels and their cytotoxicity effects at high concentration. Nanotechnology 19, 415102 (2008).

40. Warner, J. H., Dunlop, H. R. \& Tilley, R. D. Surface morphology dependent photoluminescence from colloidal silicon nanocrystals. J. Phys. Chem. B 109, 19064-19067 (2005).

41. Lin, S. W. \& Chen, D. H. Synthesis of water-soluble blue photoluminescent silicon nanocrystals with oxide surface passivation. Small 5, 72-76 (2009).

42. Holmes, J. D. et al. Highly luminescent silicon nanocrystals with discrete optical transitions. J. Am. Chem. Soc. 123, 3743-3748 (2001).

43. Wilcoxon, J. P., Provencio, P. P. \& Samara, G. A. Optical and electronic properties of Si nanoclusters synthesized in inverse micelles. Phys. Rev B. 60, 2704-2714 (1999).

44. Zhou, Z., Brus, L. \& Friesner, R. Electronic structure and luminescence of 1.1- and 1.4-nm silicon nanocrystals: Oxide shell versus hydrogen passivation. Nano Lett. 3, 163-167 (2003).

45. Rosenthal, S. J. et al. Targeting cell surface receptors with ligand-conjugated nanocrystals. J. Am. Chem. Soc. 124, 4586-4594 (2002)

46. Jaiswal, J. K., Mattoussi, H., Maruro, J. M. \& Simon, S. M. Long-term multiple color imaging of live cells using quantum dot bioconjugates. Nat. Biotechnol. 21, 47-51 (2003).

47. Wu, X. et al. Immunofluorescent labeling of cancer marker Her2 and other cellular targets with semiconductor quantum dots. Nat. Biotechnol. 21, 41-46 (2003).

48. Mattoussi, H. et al. Self-assembly of $\mathrm{CdSe}-\mathrm{ZnS}$ quantum dot bioconjugates using an engineered recombinant protein. J. Am. Chem. Soc. 122, 12142-12150 (2000).

49. Yang, C. S. et al. Synthesis of alkyl-terminated silicon nanoclusters by a solution route. J. Am. Chem. Soc. 121, 5191-5195 (1999).
50. Pettigrew, K. A., Liu, Q., Power, P. P. \& Kauzlarich, S. M. Solution synthesis of alkyl- and alkyl/alkoxy-capped silicon nanoparticles via oxidation of $\mathrm{Mg}_{2} \mathrm{Si}$. Chem. Mater. 15, 4005-4011 (2003).

51. Erogbogbo, F. et al. Bioconjugation of luminescent silicon quantum dots for selective uptake by cancer cells. Bioconjug. Chem. 22, 1081-1088 (2011).

52. Hu, D. et al. A fast synthesis of near-infrared emitting CdTe/CdSe quantum dots with small hydrodynamic diameter for in vivo imaging probes. Nanoscale 3, 47244732 (2011).

53. Sun, H. T. et al. Highly fluorescent silica-coated bismuth-doped aluminosilicate nanoparticles for near-infrared bioimaging. Small 7, 199-203 (2011).

54. Zhai, C. et al. One-pot synthesis of biocompatible CdSe/CdS quantum dots and their applications as fluorescent biological labels. Nanoscale Res. Lett. 6, 31 (2011)

55. Guiducci, C. et al. Properties regulating the nature of the plasmacytoid dendritic cell response to Toll-like receptor 9 activation. J. Exp. Med. 203, 1999-2008 (2006).

56. Sasai, M., Linehan, M. M. \& Iwasaki, A. Bifurcation of Toll-like receptor 9 signaling by adaptor protein 3. Science 329, 1530-1534 (2010).

57. Honda, K., Ohba, Y., Yanai, H., Negishi, H., Mizutani, T., Takaoka, A., Taya, C. \& Taniguchi T. Spatiotemporal regulation of MyD88-IRF-7 signaling for robust type-I interaction. Nature 434, 1035-1040 (2005).

58. Hass, T., Schmitz, F., Heit, A. \& Wagner, H. Sequence independent interferon- $\alpha$ induction by multimerized phosphodiester DNA depends on spatial regulation of Toll-like receptor activation in plasmacytoid dendritic cells. Immunology 126, 290-298 (2008)

59. Liu, Y. C., Gray, R. C., Hardy, G. A. D., Kuchtey, J., Abbott, D. W., Emancipator, S. N. \& Harding, C. V. CpG-B oligodeoxynucleotides inhibit TLR-dependent and -independent induction of type I IFN in dendritic cells. J. Immunol. 184, 33673376 (2010).

60. Warner, J. H., Hoshino, A., Yamamoto, K. \& Tilley, R. D. Water-Soluble Photoluminescent Silicon Quantum Dots. Angew. Chem. Int. Ed. 44, 4550-4554 (2005).

\section{Acknowledgments}

We thank Dr. S.A. Chechetka and Ms. S. Kajiwara for technical assistance. This work was supported by a Grant-in-Aid for Scientific Research (C-22560777 and 23-01510) from the Japan Society for the Promotion of Science and the Ministry of Education, Culture, Sports, Science and Technology (MEXT) to NH, and by the Nanotechnology Network Program Japan from MEXT.

\section{Author contributions}

S.Chinnathambi contributed to most of the experiments, including the preparation and characterization of Si-NPs and the cytotoxicity and cytokine assays. S.Chen and SG participated in the preparation and characterization of Si-NPs, respectively. $\mathrm{NH}$ designed and supervised the study, analyzed the data, and wrote the manuscript.

\section{Additional information}

Supplementary information accompanies this paper at http://www.nature.com/ scientificreports

Competing financial interests: The authors declare no competing financial interests.

License: This work is licensed under a Creative Commons

Attribution-NonCommercial-NoDerivative Works 3.0 Unported License. To view a copy of this license, visit http://creativecommons.org/licenses/by-nc-nd/3.0/

How to cite this article: Chinnathambi, S., Chen, S., Ganesan, S. \& Hanagata, N. Binding Mode of CpG Oligodeoxynucleotides to Nanoparticles Regulates Bifurcated Cytokine induction via Toll-like Receptor 9. Sci. Rep. 2, 534; DOI:10.1038/srep00534 (2012). 